\title{
Confocal microphotoluminescence of InGaN-based light-emitting diodes
}

\section{$\operatorname{AUTHOR}(\mathrm{S})$ :}

Okamoto, K; Kaneta, A; Kawakami, Y; Fujita, S; Choi, J; Terazima, M; Mukai, T

\section{CITATION:}

Okamoto, K ...[et al]. Confocal microphotoluminescence of InGaNbased light-emitting diodes. JOURNAL OF APPLIED PHYSICS 2005, 98(6): 064503.

\section{ISSUE DATE:}

2005-09-15

URL:

http://hdl.handle.net/2433/50155

\section{RIGHT:}

Copyright 2005 American Institute of Physics. This article may be downloaded for personal use only. Any other use requires prior permission of the author and the American Institute of Physics. 


\title{
Confocal microphotoluminescence of InGaN-based light-emitting diodes
}

\author{
Koichi Okamoto, ${ }^{\text {a) }}$ Akio Kaneta, Yoichi Kawakami, and Shigeo Fujita \\ Department of Electronic Science and Engineering, Kyoto University, Kyoto 615-8510, Japan \\ Jungkwon Choi and Masahide Terazima \\ Department of Chemistry, Kyoto University, Kyoto 606-8502, Japan \\ Takashi Mukai \\ Nitride Semiconductor Research Laboratory, Nichia Corporation, 491 Oka, Kaminaka, Anan, \\ Tokushima 774-8601, Japan
}

(Received 25 June 2004; accepted 28 July 2005; published online 19 September 2005)

\begin{abstract}
Spatially resolved photoluminescence (PL) of InGaN/GaN/AlGaN-based quantum-well-structured light-emitting diodes (LEDs) with a yellow-green light $(530 \mathrm{~nm})$ and an amber light $(600 \mathrm{~nm})$ was measured by using confocal microscopy. Submicron-scale spatial inhomogeneities of both PL intensities and spectra were found in confocal micro-PL images. We also found clear correlations between PL intensities and peak wavelength for both LEDs. Such correlations for yellow-green and amber LEDs were different from the reported correlations for blue or green LEDs. This discrepancy should be due to different diffusion, localization, and recombination dynamics of electron-hole pairs generated in InGaN active layers, and should be a very important property for influencing the optical properties of LEDs. In order to explain the results, we proposed a possible carrier dynamics model based on the carrier localization and partial reduction of the quantum confinement Stark effect depending on an indium composition in InGaN active layers. By using this model, we also considered the origin of the reduction of the emission efficiencies with a longer emission wavelength of InGaN LEDs with high indium composition. () 2005 American Institute of Physics. [DOI: $10.1063 / 1.2037869$ ]
\end{abstract}

\section{INTRODUCTION}

Currently, InGaN/GaN quantum-well- (QW) structured light-emitting diodes (LEDs) with several visible spectral regions have been fabricated and used widely. ${ }^{1-4}$ At the blue spectrum region, the external quantum efficiency $\left(\eta_{\mathrm{ext}}\right)$ of the LED has achieved $20 \%$ at room temperature in spite of high threading dislocation (TD) density $\left(10^{8}-10^{10} \mathrm{~cm}^{-2}\right)$. Moreover, a white light LED, in which a blue LED is combined with a yellow phosphor, has been commercialized and offers a replacement for conventional incandescent and fluorescent light bulbs. ${ }^{5}$ However, luminous efficacies of commercial white LEDs $(25 \mathrm{~lm} / \mathrm{W})$ have been still lower than that of fluorescent tubes $(75 \mathrm{~lm} / \mathrm{W})$. Thus, the most important requirement for competitive LEDs for solid-state lighting is the enhancement of quantum efficiency of emission. In order to achieve such development, we have to elucidate and understand the important factors that determine the optical properties of InGaN LEDs.

Recently, it has become known that microscopic inhomogeneities of the optical properties of InGaN active layers are very important for emission efficiencies. ${ }^{6-8}$ The microscopic spatial inhomogeneities of the optical properties should be generated by a fluctuation of indium composition by a phase separation in InGaN layers. The carriers, created by electron injection or photoexcitation, are localized in small volumes of indium-rich areas in $\mathrm{InGaN}^{6-8}$ and hin-

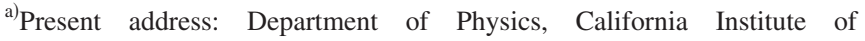
Technology, MC 136-93, Pasadena, CA 91125, USA; electronic mail: kokamoto@caltech.edu
}

dered to be trapped in nonradiative recombination centers (localization model). ${ }^{9,10}$ Another important feature of the emission mechanics of $\mathrm{InGaN} / \mathrm{GaN}$ is the quantum confinement Stark effect ${ }^{11-13}$ (QCSE) which results from strong piezoelectric fields $\left(F_{\mathrm{PZ}}\right)$ in strained InGaN QWs. ${ }^{14,15}$ Strain induces change in the energy-band diagrams of InGaN separating electron-hole pairs and decreasing the emission efficiencies. The QCSE becomes more effective with an increasing indium composition in InGaN QWs. Avoiding QCSE is crucially important for higher performance LEDs. Recently, $F_{\mathrm{PZ}}$ values across the $\mathrm{QW}$ of the order of $1.4 \mathrm{MV} / \mathrm{cm}$ were confirmed in amber InGaN single QW LEDs. ${ }^{16}$ Local profiles of the QCSE due to the spatial inhomogeneity of the indium composition are also expected to be a very important factor of the optical properties.

While a microscopic photoluminescence (PL) spectroscopy has been used to characterize such microscopic optical properties, the spatial resolutions of micro-PL were limited by the diffraction limit of the light wave. Recently, a cathodoluminescence ${ }^{17-19}$ (CL) or a scanning near-field optical microscopy ${ }^{20-25}$ (SNOM) has been used as a powerful alternative method to obtain optical images of InGaN/GaN in a submicrometer scale. However, spatial resolutions of CL and SNOM are still limited by the diffusion lengths of carriers. At room temperature, carrier diffusion length becomes large and the spatial resolutions of the CL and SNOM become low, though the optical properties of such materials at room temperature are much more important than those at low temperatures for applications to optical devices.

Quite recently, we have reported the observation of the 
submicron-scale PL images and spectra of an InGaN/GaN-based LED wafer by using confocal microscopy. ${ }^{26}$ A confocal optical system enables a highly spatial resolution beyond the diffraction limit of a light wave. ${ }^{27,28}$ Previously, confocal microscopy has been used to measure spatially resolved Raman spectroscopy to characterize the stresses ${ }^{29,30}$ and inhomogeneities of the yellow band emission $^{31}$ of a GaN crystal. O'Donnell et al. have published confocal microscopic images of $\mathrm{InGaN} / \mathrm{GaN}$ with a much larger scale. ${ }^{32,33}$ We have showed that confocal microscopy is a very powerful and convenient technique to observe the submicron-scale optical properties of InGaN-based LED and other optical devices. ${ }^{26}$

In this paper, we focus our attention to the confocal micro-PL measurement of InGaN-based LEDs with high indium composition, which has yellow-green or amber wavelength emission. One of the problems of the InGaN-based LED is the remarkable reduction of the emission efficiency of InGaN with high indium composition. ${ }^{3,4}$ The light output of the InGaN-based LED with yellow-green, yellow, or red wavelength emission has been very dark, although the reason for this is still unknown. Until now, there have been many reports about the spatially resolved PL studies for InGaNbased LEDs with bright blue or green emission. However, similar studies for LEDs with high indium compositions so far have not been studied in spite of the importance for wider applications of LEDs. Here, we measured the spatial profiles of the optical properties of LEDs with yellow-green or amber emission. We compared the results with the previous studies for LEDs with blue emission and considered the origin of the reduction of the emission efficiencies of InGaN LEDs with high indium compositions.

\section{EXPERIMENT}

The samples of InGaN/GaN/AlGaN-based LED wafers with yellow-green light and amber light were grown by a metal-organic chemical-vapor deposition (MOCVD) method on a (0001) $C$-face sapphire substrate. The device structures consisted of a $30-\mathrm{nm}$ GaN buffer layer, a 5- $\mu$ m-thick layer of $n$-type $\mathrm{GaN}: \mathrm{Si}$, a 3-nm-thick active layer of undoped $\mathrm{In}$ $\mathrm{GaN}$, a 60 -nm-thick layer of $p$-type AlGaN:Mg, and a 150 -nm-thick layer of $p$-type GaN:Mg.

The PL spectra of InGaN-based LED with yellow-green and amber emissions are shown in Fig. 1(a). For reference, PL spectrum of the same structured LED wafers with blue emission is also shown together. The PL peak wavelengths of blue, yellow-green, and amber LEDs are 480, 530, and $600 \mathrm{~nm}$, respectively. From these peak wavelengths, the indium compositions of blue, yellow-green, and amber LEDs are roughly estimated to $15 \%, 30 \%$, and $45 \%$, respectively by using the indium-composition-dependent PL spectra. ${ }^{34}$ $\eta_{\text {ext }}$ values of yellow-green LED and amber LED driven under $20 \mathrm{mV}$ were reported as $7 \%$ and $3 \%$, respectively. ${ }^{3,4}$ The PL lifetimes of yellow-green and amber samples are reported as 16 and $28 \mathrm{~ns}$, respectively, by the time-resolved PL measurement. ${ }^{35}$ Figure 1 (b) shows the Arrhenius plots of the integrated PL intensities for blue, yellow-green, and amber LEDs. The integrated PL intensities are constant in a low-
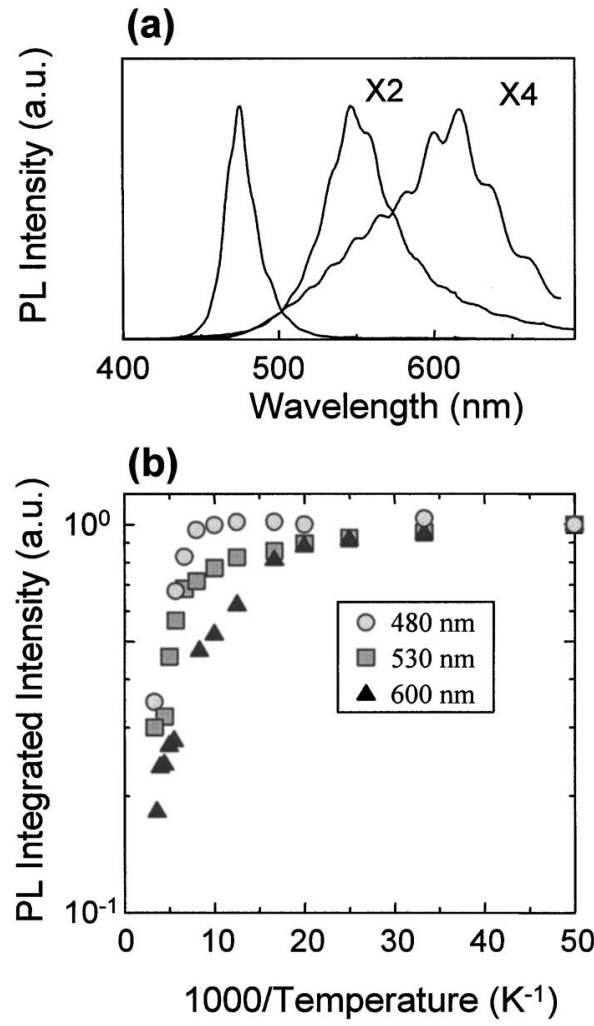

FIG. 1. (a) PL spectra of the InGaN/GaN/AlGaN-based LED wafers with blue, yellow-green, and amber light emissions. (b) Arrhenius plots of the PL intensities integrated over the emission spectra. PL-integrated intensities at $20 \mathrm{~K}$ were normalized to 1 .

temperature region, and then decrease gradually with increasing temperature. In such a case, the internal quantum efficiency $\left(\eta_{\text {int }}\right)$ of emission can be estimated by assuming that $\eta_{\text {int }}$ is nearly equal to unity at low temperature $(20 \mathrm{~K})$. $\eta_{\text {int }}$ are estimated to be $35 \%, 30 \%$, and $18 \%$ for blue, yellowgreen, and amber LEDs, respectively.

The experimental setup of a confocal microscopy is shown in Fig. 2. A confocal microscopy system manufac-

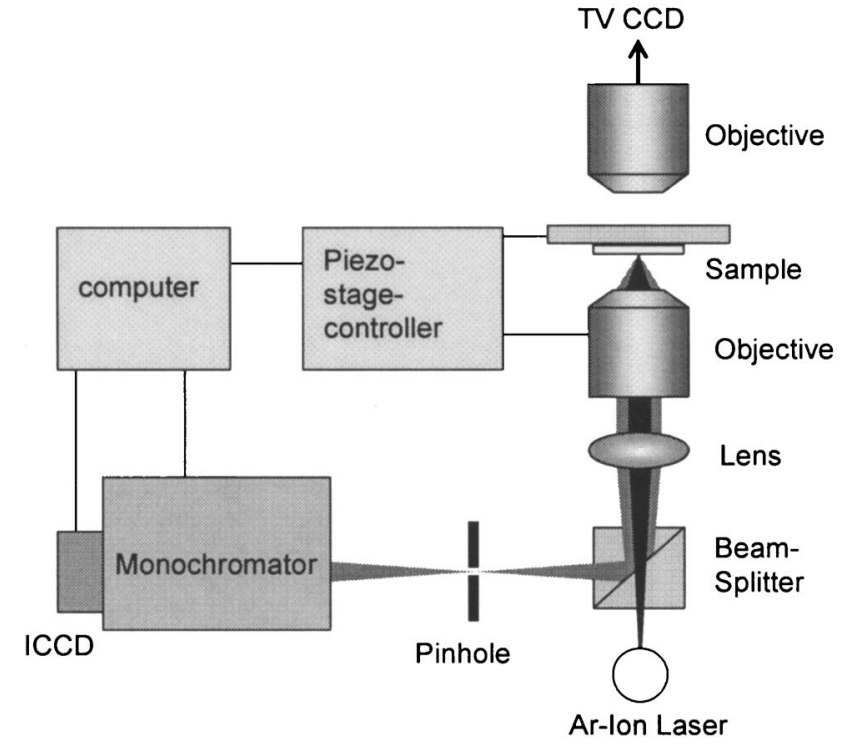

FIG. 2. Experimental setup of the confocal micro-PL measurement. 


\section{Excitation Laser}

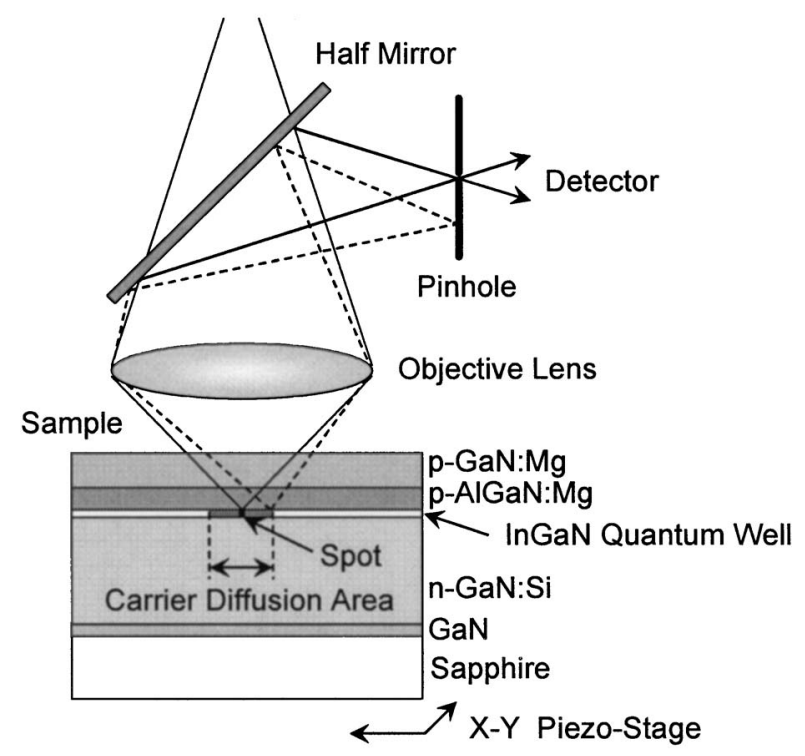

FIG. 3. Optical configuration of the confocal microscope and the sample structure of the InGaN-based LED wafer.

tured by Tokyo Instrument (Nanofinder) ${ }^{36}$ is used with a $\times 100$ objective lens [numerical aperture $\mathrm{NA}=0.95, F$ $=1.8 \mathrm{~mm}]$. A cw beam from a 488-nm line of an $\mathrm{Ar}^{+}$laser at $0.5 \mathrm{~mW}$ is used as the excitation beam. The focal size of the laser beam is $300 \mathrm{~nm}$ under these conditions. PL signals emitted from the samples are focused again on a small pinhole $(8.5 \mu \mathrm{m})$ and the signals that passed through this pinhole are detected using a charge-coupled device (CCD) detector with a monochromator. The lateral spatial resolution of this system is about $200 \mathrm{~nm} .^{36}$ The measurement is performed at room temperature $\left(23^{\circ} \mathrm{C}\right)$.

\section{RESULT AND DISCUSSION}

\section{A. Confocal microscopic images}

Figure 3 shows the optical configuration of the confocal microscopy and the sample structure of InGaN-based LED. The excitation laser was focused around the upper layer region which includes the InGaN active layer of the LED structure. Carriers were created within an excitation spot size $(300 \mathrm{~nm})$ and diffused within the carrier diffusion length [200-600 nm for InGaN/GaN (Refs. 37-39)]. PL was emitted by carrier recombination within the carrier diffusion length. Even if the excitation spot sizes of CL and SNOM were on the nanometer scale, the obtained optical images were broadened with an increasing carrier diffusion length. Using confocal microscopy, the PL emitted within the spot size can pass the pinhole and be detected. On the other hand, the PL emissions which came from outside the excitation spots cannot pass the pinhole and cannot be detected.

Figures 4 and 5 show the confocal microscopic images of the PL intensity mapping (a) and the PL peak wavelength mapping (b) by scanning a $5 \times 5-\mu \mathrm{m}^{2}$ area taken for InGaNbased LEDs with yellow-green and amber emissions, respectively. We found fine islandlike structures within the range of approximately $0.2-1 \mu \mathrm{m}$ in both PL intensities and peak wavelength. The relative PL intensity was fluctuated from 10 to 30 and the peak wavelength was fluctuated from 515 to $535 \mathrm{~nm}$ for yellow-green LED, while the relative PL intensity was fluctuated from 5 to 17 and the peak wavelength was fluctuated from 590 to $630 \mathrm{~nm}$ for the amber LED. From the clearness of the fine structures in these images, the spatial resolution was estimated at about $200 \mathrm{~nm}$, which was much smaller than the carrier diffusion length at room temperature $(\sim 600 \mathrm{~nm}){ }^{39}$ These high spatially resolved PL images should be attributed to the advantage of the confocal microscopy, i.e., the diffraction-limit-free and the detection areas smaller than the carrier diffusion length. Such clear images have not been obtained at room temperature by a usual microscope, even when using the CL measurement at room temperature ${ }^{40,41}$ or SNOM with illumination or collection operation. ${ }^{20-22}$ Quite recently, our group succeeded in obtaining the highly resolved PL images by using the SNOM with illumination-collection mode. ${ }^{23,24}$ In this method, both excitation and detection were operated through the same optical fiber tip. The similar highly resolved PL image is obtainable by using the confocal microscopy, while the experimental setup and operation are much easier.

The observed submicron-scaled PL inhomogeneities should be due to the spatial fluctuation of the energy band gap, which is mainly created by the spatial distribution of indium composition in InGaN active layers. We found that the correlation between the PL intensity [Fig. 4(a)] and peak wavelength [Fig. 4(b)] of the yellow-green LED was not so clear. We have already reported the similar behavior of yellow-green LED probed by the SNOM measurement ${ }^{23}$ and confocal microscopy. ${ }^{26}$ In this case, the areas of long PL wavelength (small PL peak energy) correspond to indiumrich areas. On the other hand, we can notice much clearer correlation between Figs. 5(a) and 5(b) for the amber LED. The points with strong PL intensities have shorter PL wavelengths, while the points with weak PL intensities have longer PL wavelengths. In our recent report, a clear correlation has been reported between the PL intensity and the wavelength in spatially resolved PL images of blue LEDs by using SNOM. ${ }^{24}$ In the case of blue LED, the areas of strong PL intensity correspond to those at long PL wavelength. The tendencies of PL intensity-peak correlations observed for the yellow-green and amber LEDs were quite different. This discrepancy should be due to the different behaviors of the carrier dynamics in active layers. The detailed relationship between the PL properties and the carrier dynamics will be discussed in the later section.

\section{B. Submicron-scale PL spectra}

Figure 6 shows the submicron-scale PL spectra of (a) yellow-green and (b) amber LEDs at positions A-E marked in Figs. 3 and 4, respectively. We obtained the PL spectra with various intensities and peak wavelengths at each position. The spatial resolution of these spectra is about $200 \mathrm{~nm}$. The obtained PL spectra have a broad linewidth, which are about $30 \mathrm{~nm}(135 \mathrm{meV})$ for the yellow-green emission and $50 \mathrm{~nm}(175 \mathrm{meV})$ for the amber emission. These values of 

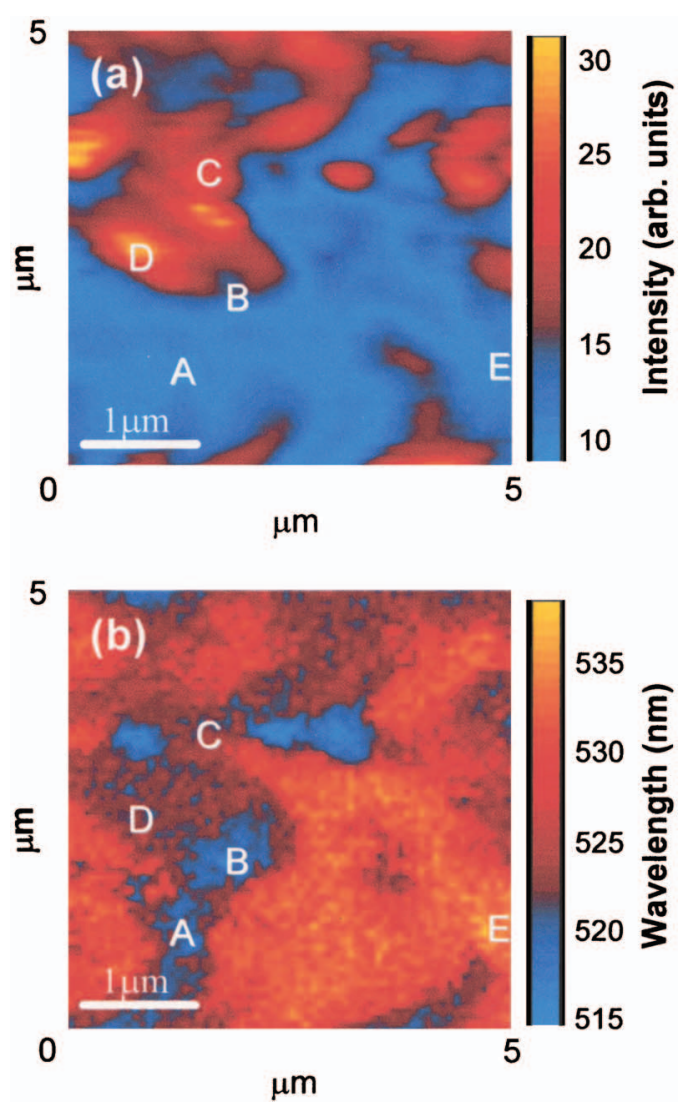

FIG. 4. (Color) Peak intensity mapping (a) and peak wavelength mapping (b) of the PL by scanning a $5 \times 5-\mu \mathrm{m}^{2}$ area using the confocal microscope taken for InGaN-based LED with yellow-green emission at room temperature.

linewidth are smaller than those of the macroscopic spectra shown in Fig. 1(a). However, these values are much larger than the reported PL linewidth of the blue LED (11.6 meV) probed by the SNOM with $30-\mathrm{nm}$ spatial resolution at $18 \mathrm{~K}^{24}$ The broadening of the obtained PL spectra of yellow-green and amber emissions should be due to the inhomogeneous broadening. PL spatial inhomogeneities smaller than the spatial resolution $(200 \mathrm{~nm})$ of our confocal microscope may exist. Such smaller structures should act as the localization center of the carriers or electron-hole pair in the active layers. Another possible reason for the broad spectra is the thermal effect. The reported narrow linewidth $(11.6 \mathrm{meV})$ was measured at low temperature $(18 \mathrm{~K})$, while our results were measured at room temperature. The thermal energy of the carriers at room temperature $(26 \mathrm{meV})$ may be larger than the localization energy in active layers. Thus, many carriers are not localized in active layers at room temperature. For both reasons, our obtained spectra have a broad linewidth.

Figure 7 shows the relationship between the PL peak intensities and wavelength at 100 arbitrarily selected points including positions A-E in Figs. 4 and 5. The PL peak intensity-wavelength correlation of blue LEDs obtained previously by SNOM measurement ${ }^{24}$ was also plotted in Fig. 7(a). In this figure, a clear increase along the wavelength was obtained. The tendency has been reported by Itoh $e t$ al. for LEDs in the green wavelength region around $510 \mathrm{~nm}$ by us-
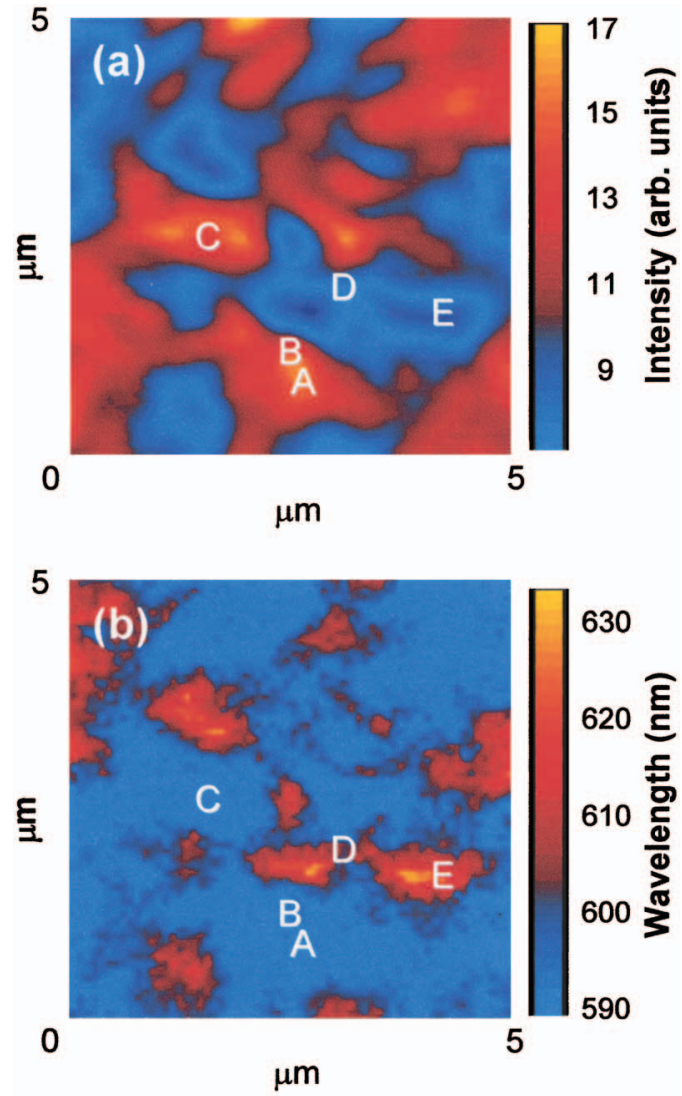

FIG. 5. (Color) Peak intensity mapping (a) and peak wavelength mapping (b) of the PL by scanning a $5 \times 5-\mu \mathrm{m}^{2}$ area using the confocal microscope taken for InGaN-based LED with amber emission at room temperature.

ing SNOM measurement with electrical pumping. ${ }^{25}$ Such PL peak intensity-wavelength correlation has been well interpreted by using the carrier localization model. The created carriers diffuse and localize into the minimum-energy region, which is attributed to the fluctuations of the indium composition in the InGaN active layers. Quite recently, the spatially localized dynamics of carriers in InGaN have been observed directly by using the time-resolved SNOM measurement. ${ }^{42}$ Such carrier localization dynamics is a very important feature of InGaN in the low indium composition regions (bluegreen emission). This localization process should be the main origin for the high emission efficiency of blue or green LEDs.

Here, we found a quite different behavior of the PL peak intensity-wavelength correlation of yellow-green and amber LEDs in Fig. 7. The correlation of yellow-green LED looked normally distributed [Fig. 7(b)], while that of amber LED seemed to be simply decreasing along the wavelength [Fig. 7(c)]. The correlation of amber LED is quite opposite to that reported for blue or green LEDs. ${ }^{24,25}$ This may suggest that the lower-energy regions in the active layer of amber LED should not be as effective as the localization center of the carriers. The critical point on the wavelength of these two types of correlations is around $520 \mathrm{~nm}$, as shown in Fig. 7(b). At the wavelength regions shorter than $520 \mathrm{~nm}$, the PL intensities become larger with an increasing wavelength. At the wavelength regions longer than $520 \mathrm{~nm}$, the PL intensities become smaller with the increasing of wavelength. This 

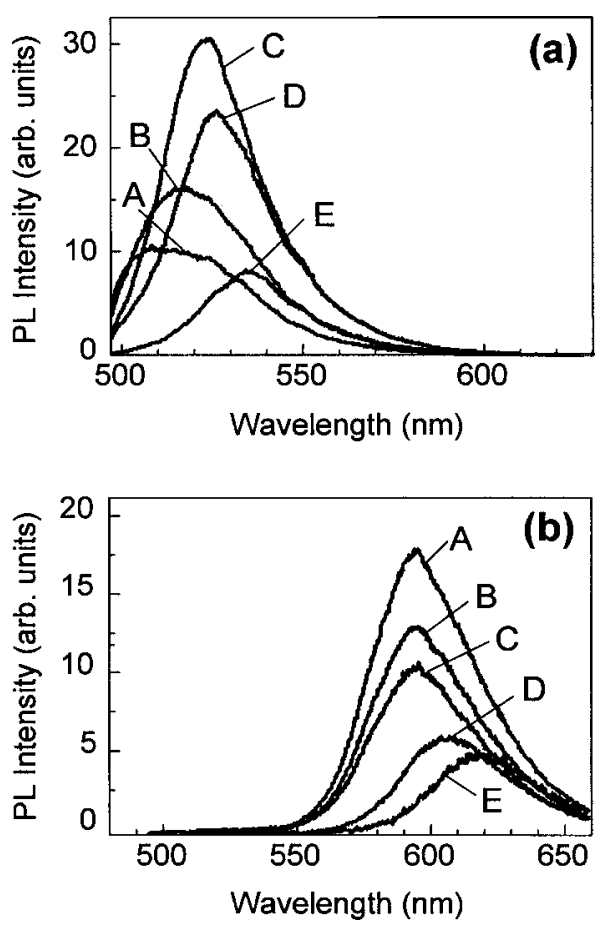

FIG. 6. Spatially resolved PL spectra of InGaN-based LEDs with yellowgreen emission (up) and amber emission (down) at room temperature at positions A-E marked in Figs. 4 and 5.

tendency should be related to the reduction of emission efficiencies of InGaN LEDs with high indium composition.

\section{Dynamics model of electron-hole pair}

In order to explain the results, we considered the possible dynamics and mechanism by using both carrier localization model and QCSE model. Figure 8 shows the indiumcomposition-dependent diffusion, localization, and recombination dynamics of electron-hole pairs in InGaN active layers. Both the conduction- and valence-band structures of InGaN active layers should be spatially fluctuated due to the spatial distribution of the indium composition. Figure 8(a) shows the band structure under low indium doping (blue or green LED). In this case, indium composition is lower (around a few tens percent) than the gallium composition, and the indium-rich areas are quite smaller than the indiumpoor areas in $\mathrm{InGaN}$. Then, the spatial fluctuations of the indium-rich areas can act as the localization center of carriers, and the probability of the radiative recombination of electron-hole pairs becomes higher at these areas (emission centers). The delocalized carriers in the indium-poor areas have a low probability of radiative recombination and migrate to the indium-rich areas or go to the nonradiative center to be lost as heat. This is well known as carrier localization model.

On the other hand, the band structure under the high indium composition (yellow or amber LED) should be different. In this case, indium composition is as high as the gallium composition, and the indium-rich areas become as large as in size comparable to the indium-poor areas, while become much larger than low indium-doped InGaN as shown in Fig. 8(b). Then, the carriers in indium-rich areas
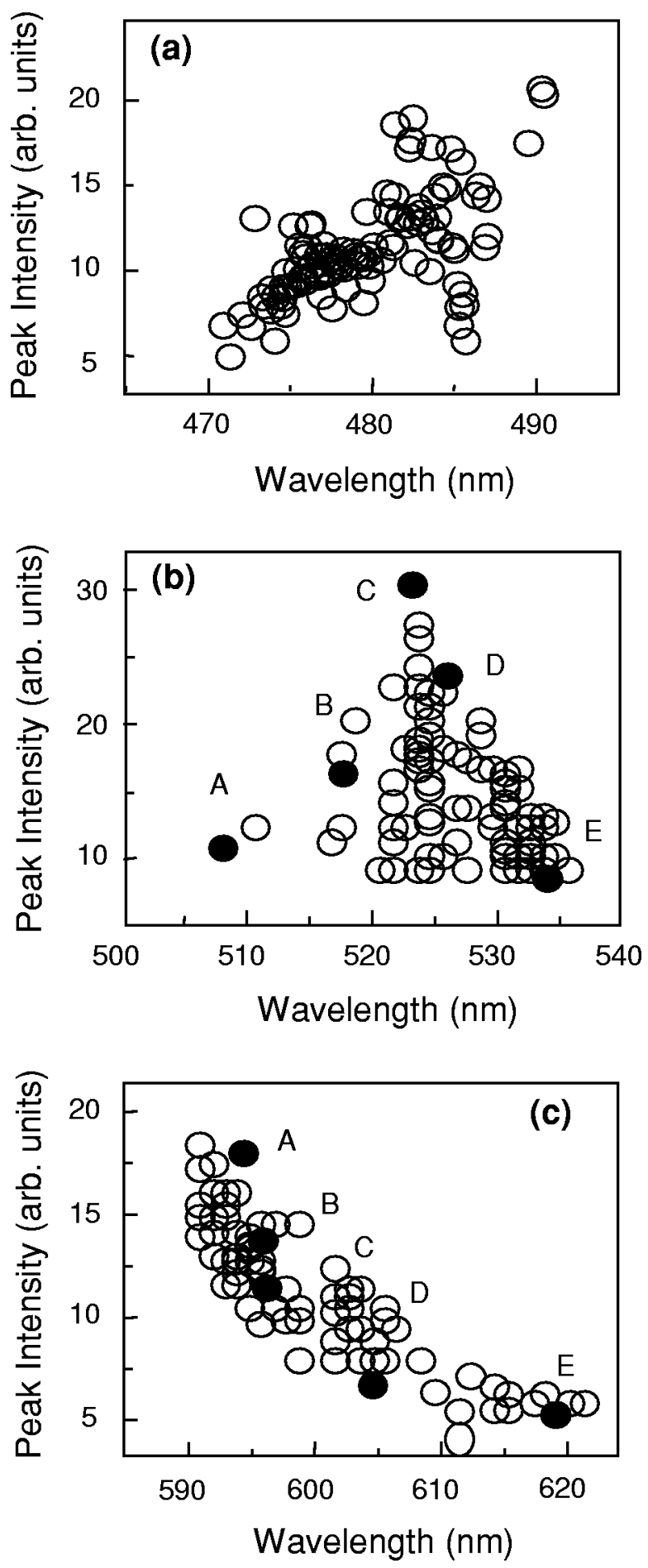

FIG. 7. Correlation between the PL peak wavelength and intensity at 100 arbitrarily chosen points of InGaN-based LEDs with (a) blue emission (measured by SNOM in Ref. 24), (b) yellow-green emission in Fig. 4, and (c) amber emission in Fig. 5.

are not localized and can diffuse easily. Thus, indium-rich areas should not act as the emission centers and the probability of radiative recombination becomes lower in the $\mathrm{InGaN}$ active layers.

Moreover, the QCSE effect should be dominant in amber LEDs. It reduces the electron-hole wave-function overlap, decreases the probability of radiative recombination [Fig. 8(c)], and increases in PL lifetime. In the indium-rich areas, the internal electric fields are screened by the higher densi- 

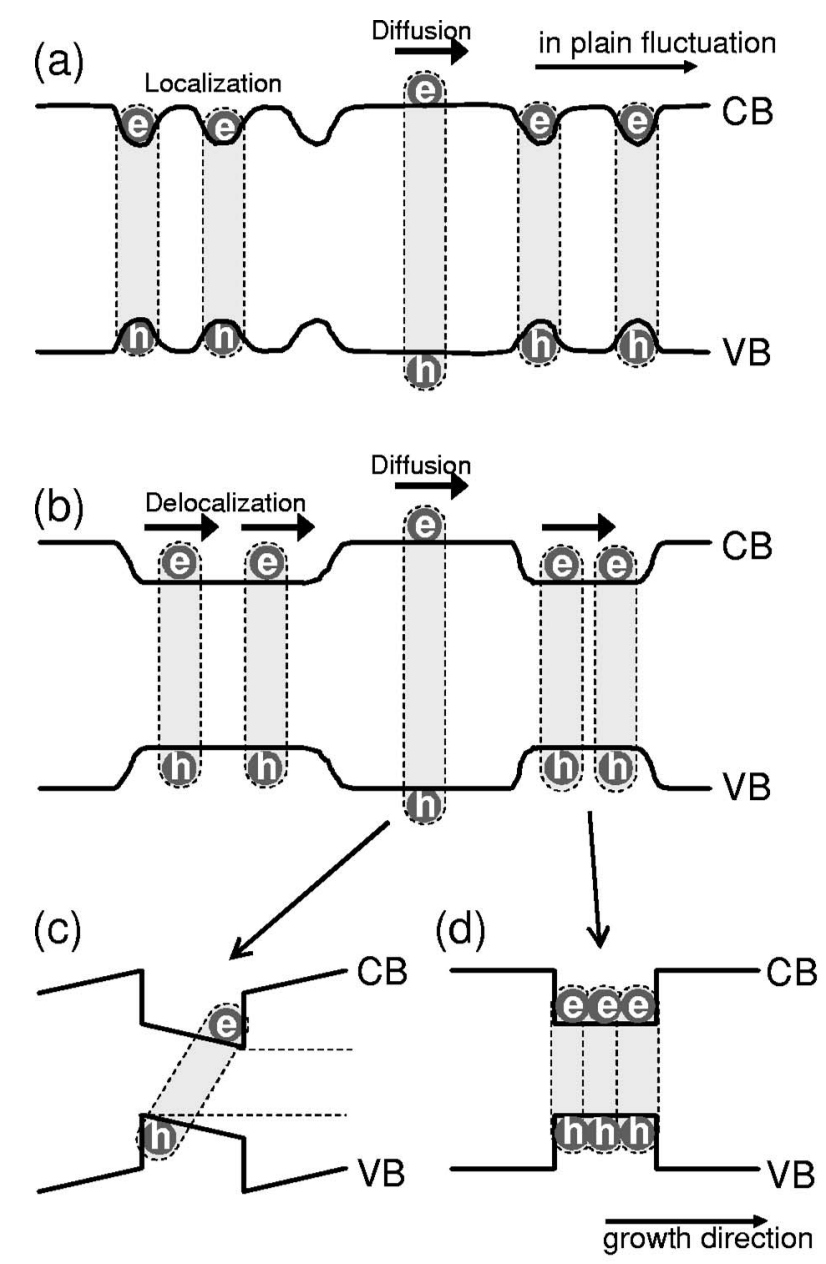

FIG. 8. Schematic band diagrams of in-plane fluctuation and diffusion, localization, and recombination dynamics of electron-hole pairs in InGaN active layers which have (a) low indium composition (blue or green emission) and (b) high indium composition (amber emission). (c) Schematic band diagram strained by strong piezoelectric field along the growth direction. (d) Partial reduction of QCSE by bond filling at the high-density regions of generated electron-hole pairs.

ties of generated carriers. Thus, QCSE is reduced [Fig. 8(d)]. The spatial variation of the measured PL peak wavelength [Fig. 5(b)] can be attributed to the blueshift of the PL peak by the partial screening of the QCSE. In this case, the areas of short PL wavelength correspond to those of high carrier density (indium-rich) areas. This can explain the opposing PL intensity-peak correlations of InGaN with high indium compositions. If the $F_{\mathrm{PZ}}$ in the indium-rich areas is screened and the effective band gap becomes larger than that of the surrounding areas, carriers may diffuse away from the indium-rich areas. Previously, it was reported that the $F_{\mathrm{PZ}}$ screening increases recombination rates. ${ }^{16}$ Recently, Walterelt et al. developed piezoelectric-field-free GaN/AlGaN QW grown on the $M$ plane of a GaN substrate and observed about ten-times faster PL decay. ${ }^{43}$ Such fast recombination rates in screened areas may prevent carrier diffusion from the indium-rich areas.

Similar partial $F_{\mathrm{PZ}}$ screening effect may contribute to blue LEDs with lower indium composition. Recently, Omae et al. succeeded in separating the localization and internal electric-field effects by transient absorption spectra probed with an ultrafast pump and probe measurement. ${ }^{44,45}$ It was found that the QCSE depends on the QW thickness and indium composition, and only the localization effect was observed in blue InGaN/GaN with $>5-\mathrm{nm}$ QW thickness. ${ }^{45}$ Therefore, we conclude that the localization effect is dominant for our blue LEDs, whereas the QCSE is dominant for amber LEDs. The critical point of each effect was observed for the yellow-green LED around $520 \mathrm{~nm}$.

The different behaviors of localization and QCSE dynamics of electron-hole pairs in InGaN active layers should be the reason for different optical properties with several indium compositions. The delocalization of carriers in larger indium-rich areas and reducing the electron-hole wavefunction overlapping by the QCSE should be the main reason of the reduction of the emission efficiencies of LEDs with a longer emission wavelength. This reduction has been often explained by the low crystal quality of the high indium composition and activation of the nonradiative recombination centers. However, recently we have found that the nonradiative recombination lifetime $\left(\tau_{\text {non }}\right)$ of electron-hole pairs in amber LED $\left(\tau_{\text {non }}=35 \mathrm{~ns}\right)$ is longer than that in yellow-green LED $\left(\tau_{\text {non }}=23 \mathrm{~ns}\right) .{ }^{35}$ This means that the nonradiative recombination processes in amber LED are not as active as in yellow-green LED. We have also found that the radiative recombination lifetime $\left(\tau_{\text {rad }}\right)$ in amber $\operatorname{LED}\left(\tau_{\text {rad }}=156 \mathrm{~ns}\right)$ is much slower than that in yellow-green LED $\left(\tau_{\text {rad }}=51 \mathrm{~ns}\right){ }^{35}$ This suggests that the reason for the low efficiencies of InGaN LEDs with high indium composition is not due to the increasing of the nonradiative recombination, but due to the decreasing of the radiative recombination by the remarkable QCSE in the InGaN active layer. Such a difference of the carrier dynamics should be attributed to the different spatial inhomogeneities and indium compositions of InGaN active layers. The submicron-scale spatial inhomogeneity of the optical properties observed here is a very important factor but has been very difficult to observe experimentally. The confocal microscope is a powerful technique for such submicron-scale measurement.

\section{CONCLUSION}

Submicron-scale spatial inhomogeneities of the optical properties of the InGaN/GaN/AlGaN-based LEDs with yellow-green and amber emissions were observed and compared by using a confocal microscope. We found a clear difference of correlation between PL intensities and peak wavelengths for each LED. It has been reported for blue and green LEDs that the PL intensity becomes larger with an increasing wavelength. However, we found that the PL peak intensity-wavelength correlation of amber LED was quite opposite to the reported behaviors of blue or green LEDs. The PL intensity of amber LED becomes smaller with an increasing wavelength. The correlation of yellow-green LED looked like the normal distribution shape with a maximum near $520 \mathrm{~nm}$. We explained such discrepancy by using the model based on both localization and QCSE of electron-hole pairs in InGaN. Under the low indium doping up to a few tens percent the sizes of the indium-rich areas are small and act as the localization center of carriers. But under the high 
indium composition over around 30\%, the sizes of the indium-rich areas become larger and do not act as the carrier localization center and partial QCSE becomes much effective. Such different carrier dynamics should be the reason for the reduction of the emission efficiencies of LEDs with a longer emission wavelength. We found that the various spatial distributions of the optical properties of InGaN depended on the indium composition. Based on these results, we propose that controlled artificial spatial inhomogeneity in InGaN active layer should be more effective than making the crystal quality higher by reducing the dislocation density in order to improve the emission efficiency of InGaN LEDs with high indium composition.

\section{ACKNOWLEDGMENTS}

The authors wish to thank Professor A. Scherer, G. Maltezos, and A. Shvartser of California Institute of Technology for valuable discussions and reviewing the manuscript. This work was partly supported by Japan Society for the Promotion of Science (Grant No. 00122454) and the Kyoto University-Venture Business Laboratory Project.

${ }^{1}$ S. Nakamura, T. Mukai, and M. Senoh, Appl. Phys. Lett. 64, 1687 (1994). ${ }^{2}$ S. Nakamura, T. Mukai, M. Senoh, and N. Iwase, J. Appl. Phys. 76, 8189 (1994).

${ }^{3}$ T. Mukai and S. Nakamura, Jpn. J. Appl. Phys., Part 1 38, 5735 (1999).

${ }^{4}$ T. Mukai, M. Yamada, and S. Nakamura, Jpn. J. Appl. Phys., Part 1 38, 3976 (1999).

${ }^{5}$ S. Nakamura and G. Fasol, The Blue Laser Diode: GaN Based Light Emitting and Lasers (Springer, Berlin, 1997).

${ }^{6}$ S. Chichibu, T. Azuhata, T. Sota, and S. Nakamura, Appl. Phys. Lett. 69, 4188 (1996).

${ }^{7}$ Y. Narukawa, Y. Kawakami, M. Funato, Sz. Fujita, Sg. Fujita, and S. Nakamura, Appl. Phys. Lett. 70, 981 (1997).

${ }^{8}$ Y. Narukawa, Y. Kawakami, Sz. Fujita, Sg. Fujita, and S. Nakamura, Phys. Rev. B 55, R1938 (1997).

${ }^{9}$ S. D. Lester, F. A. Fonce, M. G. Craford, and D. A. Steigerward, Appl. Phys. Lett. 66, 1249 (1995).

${ }^{10}$ T. Mukai, K. Takekawa, and S. Nakamura, Jpn. J. Appl. Phys., Part 2 37, L839 (1998).

${ }^{11}$ P. Perlin et al., Appl. Phys. Lett. 73, 2778 (1998).

${ }^{12}$ T. Takeuchi, S. Sota, M. Katsuragawa, M. Komori, H. Takeuchi, H. Amano, and I. Akasaki, Jpn. J. Appl. Phys., Part 2 36, L382 (1997).

${ }^{13}$ T. Takeuchi et al., Appl. Phys. Lett. 73, 1691 (1998).

${ }^{14}$ C. Wetzel, T. Takeuchi, S. Yamaguchi, H. Katoh, H. Amamo, and I. Akasaki, Appl. Phys. Lett. 73, 1994 (1998).

${ }^{15}$ T. Takeuchi et al., Appl. Phys. Lett. 73, 1691 (1998).

${ }^{16}$ S. F. Chichibu, T. Azuhata, T. Sota, T. Mukai, and S. Nakamura, J. Appl. Phys. 88, 5153 (2000).

${ }^{17}$ T. Sugahara, M. Hao, T. Wang, D. Nakagawa, Y. Naoi, K. Nishino, and S.
Sakai, Jpn. J. Appl. Phys., Part 2 37, L1195 (1998).

${ }^{18}$ S. Chichibu, K. Wada, and S. Nakamura, Appl. Phys. Lett. 71, 2346 (1997).

${ }^{19}$ F. Bertram, S. Srinvasan, L. Geng, F. A. Ponce, T. Riemann, and J. Christen, Appl. Phys. Lett. 80, 3524 (2002).

${ }^{20}$ D. K. Young, M. P. Mack, A. C. Abare, M. Hansen, L. A. Coldren, S. P. Denbaars, E. L. Hu, and D. Awschalom, Appl. Phys. Lett. 74, 2349 (1999).

${ }^{21}$ M. S. Jeong, J. Y. Kim, Y.-W. Kim, J. O. White, E.-K. Suh, C.-H. Hong, and H. J. Lee, Appl. Phys. Lett. 79, 976 (2001).

${ }^{22}$ M. S. Jeong, Y.-W. Kim, J. O. White, E.-K. Suh, M. G. Cheong, C. S. Kim, C.-H. Hong, and H. J. Lee, Appl. Phys. Lett. 79, 3440 (2001).

${ }^{23}$ A. Kaneta, T. Izumi, K. Okamoto, Y. Kawakami, Sg. Fujita, Y. Narita, T. Inoue, and T. Mukai, Jpn. J. Appl. Phys., Part 1 40, 110 (2001).

${ }^{24}$ A. Kaneta, K. Okamoto, Y. Kawakami, Sg. Fujita, G. Marutsuki, Y. Narukawa, and T. Mukai, Appl. Phys. Lett. 81, 4353 (2002).

${ }^{25}$ H. Itoh, S. Watanabe, M. Goto, N. Yamada, M. Misra, A. Y. Kim, and S. A. Stockman, Jpn. J. Appl. Phys., Part 2 42, L1244 (2003).

${ }^{26}$ K. Okamoto, J. Choi, Y. Kawakami, M. Terazima, T. Mukai, and Sg. Fujita, Jpn. J. Appl. Phys., Part 1 43, 839 (2004).

${ }^{27}$ N. Streibl, J. Opt. Soc. Am. A 2, 121 (1985).

${ }^{28}$ C. J. R. Sheppard, Optik (Stuttgart) 72, 13 (1986).

${ }^{29}$ M. Pophristic, F. H. Long, M. Schurman, J. Ramer, and I. T. Ferguson, Appl. Phys. Lett. 74, 3519 (1999).

${ }^{30}$ X. Li, P. W. Bohn, J. Kim, J. O. White, and J. J. Coleman, Appl. Phys. Lett. 76, 3031 (2000).

${ }^{31}$ N. V. Joshi, A. Cros, A. Cantarero, H. Medina, O. Ambacher, and M. Stutzmann, Appl. Phys. Lett. 80, 2824 (2002).

${ }^{32}$ K. P. O'Donnell, C. Trager-Cowan, S. Pereira, A. Bangura, C. Young, M. E. White, and M. J. Tobin, Phys. Status Solidi B 216, 157 (1999).

${ }^{33}$ K. P. O'Donnell, M. J. Tobin, S. C. Bayliss, and W. Van Der Stricht, J. Microsc. 193, 105 (1999).

${ }^{34}$ T. Matsuoka, H. Okamoto, M. Nakao, H. Harima, and E. Kurimoto, Appl. Phys. Lett. 81, 1246 (2002).

${ }^{35}$ K. Okamoto, S. Saijo, Y. Kawakami, Sg. Fujita, M. Terazima, T. Mukai, G. Shinomiya, and S. Nakamura, Proc. SPIE 4278, 150 (2001).

${ }^{36}$ V. A. Bykov, S. G. Feklisov, and S. A. Saunin, Phys. Low-Dimen. Struct. 3-4, 25 (2001).

${ }^{37}$ D. Cherns, S. J. Henley, and F. A. Ponce, Appl. Phys. Lett. 78, 2691 (2001).

${ }^{38}$ S. J. Henley and D. Cherns, J. Appl. Phys. 93, 3934 (2003).

${ }^{39}$ R. J. Kaplar, S. R. Kurtz, and D. D. Koleske, Appl. Phys. Lett. 85, 5436 (2004).

${ }^{40}$ B. Zhang, T. Egawa, H. Ishikawa, Y. Liu, and T. Jimbo, Jpn. J. Appl. Phys., Part 2 42, L226 (2003).

${ }^{41}$ C. Trager-Cowan, I. Osborne, M. Barisonzi, S. K. Manson-Smith, K. P. O'Donnell, K. Jacobs, I. Moerman, and P. Demeester, Phys. Status Solidi B 216, 347 (1999).

${ }^{42}$ A. Kaneta, T. Mutoh, Y. Kawakami, S. Fujita, G. Marutsuki, Y. Narukawa, and T. Mukai, Appl. Phys. Lett. 83, 3462 (2003).

${ }^{43}$ P. Walterelt, O. Brandt, A. Trampert, H. T. Grahn, J. Menniger, M. Ramsteiner, M. Reiche, and K. H. Ploog, Nature (London) 406, 865 (2000).

${ }^{44}$ K. Omae, Y. Kawakami, Sg. Fujita, M. Yamada, Y. Narukawa, and T. Mukai, Phys. Rev. B 65, 073308 (2002).

${ }^{45}$ K. Omae, Y. Kawakami, Sg. Fujita, Y. Narukawa, and T. Mukai, Phys. Rev. B 68, 085303 (2003). 